\title{
IGF-I, VEGF Y bFGF, COMO FACTORES PRONÓSTICOS DE LA APARICIÓN DE LA RETINOPATÍA DE LA PREMATURIDAD
}

\section{IGF-I, VEGF AND bFGF AS PREDICTIVE FACTORS FOR THE ONSET OF RETINOPATHY OF PREMATURITY (ROP)}

\author{
VILLEGAS-BECERRIL E ${ }^{1}$, GONZÁLEZ-FERNÁNDEZ R ${ }^{2}$, PERULA-TORRES L ${ }^{3}$, \\ GALLARDO-GALERA JM ${ }^{4}$
}

\section{RESUMEN}

Objetivo: Identificar en suero de prematuros a IGF, VEGF y bFGF, como factores de riesgo independientes en el desarrollo de la ROP. Es también nuestro objetivo el diseño de un modelo multivariante que englobe a las tres citokinas y que este pueda ser usado como parámetro indicador del examen de la ROP, además de los ya existentes.

Métodos: 74 pacientes prematuros fueron reclutados con un peso al nacer menor de 1500 grs o edad gestacional menor de 32 semanas, y fueron divididos en pacientes enfermos de ROP $(\mathrm{N}=37)$ y pacientes no enfermos de ROP $(\mathrm{N}=37)$. Se tomaron muestras de sangre de cada paciente, coincidiendo con el primer examen, entre la 4 a 6 primeras semanas de vida y estas fueron congeladas en espera de su determinación. La edad gestacional y el peso al nacer fueron evaluados.

Resultados: Encontramos diferencias significativas entre grupos en función de los valores de IGF-I y VEGF, pero no encontramos diferencias en función de la bFGF. Establecimos un modelo multivariante

\begin{abstract}
Objective: To determine if IGF-I, VEGF and bFGF, present in the serum of premature infants, are independent risk factors of the development of ROP. It was also our objective to design a multivariate model that included these three cytokines as indicator parameters in the ROP screening, in addition to the other parameters already in existence.

Methods: 74 patients were recruited with a birth weight below $1500 \mathrm{~g}$ or gestational age below 32 weeks. These were classified into those who developed ROP $(\mathrm{N}=37)$ and those without ROP $(\mathrm{N}=$ 37). We obtained serum from each infant at the time of the first examination at 4-6 postnatal weeks. These samples were frozen until the time of analysis. The roles of gestational age and birth weight were also evaluated.

Results: There were significant differences in the amount of the cytokines IGF-I and VEGF between the groups with or without ROP, but there were no significant differences for bFGF. The differences enabled us to establish a multivariate model inclu-
\end{abstract}

Recibido: 28/3/06. Aceptado: 17/11/06.

Servicio de Oftalmología. Hospital Reina Sofía de Córdoba.

1 Doctor en Medicina. Adjunto del Servicio de Oftalmología.

2 Doctor en Medicina. Adjunto del Servicio de Inmunología.

3 Doctor en Medicina.

4 Doctor en Medicina. Jefe del Servicio de Oftalmología.

Los autores manifiestan que no tienen interés comercial ni han recibido apoyo económico.

Correspondencia:

Enrique Villegas Becerril

Servicio de Oftalmología. Hospital Reina Sofía

C/. Menéndez Pidal, s/n

Córdoba

España

E-mail: drvill@terra.es 
incluyendo a la IGF-I y VEGF, para la predicción del riesgo de padecer ROP en función de estas dos citokinas.

Conclusiones: Los niveles en sangre de prematuros de estas citokinas pueden ser usados como factores indicadores del examen de la ROP, así como para predecir la probabilidad de sufrir la enfermedad.

Palabras clave: Factor de crecimiento, IGF-I, VEGF, bFGF, retinopatía de la prematuridad, citokinas. ding IGF-I and VEGF for the prediction of risk of ROP.

Conclusions: Cytokine serum levels in premature infants can be useful as an indicator in ROP screening, as well as being used to predict the probability of suffering the illness (Arch Soc Esp Oftalmol 2006; 81: 641-646).

Key words: Growth factor, IGF-I, VEGF, bFGF, retinopathy of prematurity, citokines.

\section{INTRODUCCIÓN}

En los últimos años ha crecido enormemente el interés por el mecanismo de la neovascularización ocular. Se ha postulado que la vasculogénesis y la angiogénesis son el resultado de complejas interacciones entre factores de crecimiento o mitógenos de producción tanto local como sistémica, que estimulan o inhiben la diferenciación, proliferación, migración y maduración de las células endoteliales (1). Este proceso es el responsable de enfermedades como la retinopatía diabética o la retinopatía del prematuro. En el 2003 se publica un estudio en el cual se determina el factor de crecimiento parecido a la insulina (IGF-I) en pacientes prematuros, donde concluye que no solo existe una relación entre la existencia de retinopatía de la prematuridad (ROP) y los bajos niveles de IGF-I en suero, sino que además, dichos niveles son un buen factor de predicción de la enfermedad, como los son la edad gestacional y el peso al nacer (2,3). El factor de crecimiento vascular endotelial (VEGF), es un mitógeno específico para células endoteliales, que fue identificado por diversos grupos de investigación $(4,5)$. Recientemente se le ha relacionado con la ROP mediante su cuantificación intraocular, ante repetidas fluctuaciones entre hipoxia-hiperoxia (6). Esto nos hace pensar que estas citokinas pueden ayudarnos a comprender los hallazgos clínicos observados después de dichas fluctuaciones en la ROP.

Por otra parte, se ha relacionado con este proceso vascular neoformativo al factor de crecimiento de fibroblastos básico (bFGF). Cuando se realiza la inactivación en ratones transgénicos de los genes que codifican los receptores de este factor, se provoca una fuerte inhibición de la angiogénesis retiniana (7). También los niveles de bFGF se encuentran aumentados en muestras extraídas de vítreo en pacientes con retinopatía diabética proliferativa (8). La IGF-I según la bibliografía, ha demostrado su eficacia para el diagnóstico de alteraciones en el desarrollo de la retina en pacientes prematuros $(2,3)$. El VEGF y el bFGF no han demostrado aún su uso para este objetivo, aunque se han relacionado estrechamente con la ROP mediante la experimentación en modelo animal en diversos estudios No han sido investigados sus niveles en suero aún, de manera que todavía seguimos sin conocer si pudiera existir una aplicación clínica de estas citokinas, sobre todo desde el punto de vista del diagnóstico y seguimiento de la $\operatorname{ROP}(7,9)$. Así, nuestro objetivo se fundamenta en el diseño de un modelo predictivo multivariante, del padecimiento de la ROP en función de estas tres citokinas.

\section{OBJETIVOS}

1. Estudiar el papel de las citokinas (IGF-I, VEGF y bFGF) en sangre periférica de prematuros, como posible marcador diagnóstico de la ROP.

2. Estudiar el comportamiento o interrelación de las diferentes citokinas en la etiopatogenia de la ROP, mediante un análisis multivariante que las incluya a las tres y establecer el diseño de un modelo predictivo de aparición de ROP.

3. Analizar los diferentes factores de riesgo implicados en la etiopatogenia de la enfermedad: peso al nacimiento y edad gestacional.

\section{SUJETOS, MATERIAL Y MÉTODOS}

Participantes en el estudio: se incluyeron 74 prematuros ingresados en la unidad de neonatología de nuestro centro entre octubre del 2003 y enero del 
2005. Los criterios de inclusión fueron: edad gestacional (EG) menor de 32 semanas o peso al nacer $(\mathrm{PN})<1.500$ grs. Pacientes con PN o EG mayores, con factores de riesgo para el padecimiento de la ROP o que el neonatólogo a su cargo considere que deben ser revisados, también fueron incluidos. Grupo A: $\mathrm{N}=37$, pacientes con ROP y Grupo B: $\mathrm{N}=$ 37 pacientes sin ROP. Se excluyen de este estudio todos los niños que presentan anomalías cromosómicas y alteraciones morfológicas severas. Este estudio fue aprobado por el Comité de Ética de nuestro centro. Los padres o tutores de cada paciente firmaron un consentimiento informado diseñado a este efecto.

Evaluación de la ROP: los pacientes fueron explorados entre las 4 a 6 primeras semanas de vida $(10,11)$. Se examina fondo de ojo con dilatación pupilar, (fenilefrina $2,5 \%$ y ciclopentolato $0,5 \%$ ), y oftalmoscopia binocular indirecta.

Análisis de citokinas: una única muestra de sangre venosa periférica fue recogida $(1 \mathrm{ml})$ coincidiendo con la primera exploración del despistaje de ROP, entre las 4 y 6 primeras semanas después del nacimiento. Esta fue centrifugada a $3000 \mathrm{rpm}$ y a $4^{\circ} \mathrm{C}$, siendo a continuación almacenada a $-40^{\circ} \mathrm{C}$. $\mathrm{La}$ IGF-I, VEGF Y bFGF, fueron medidas mediante los kits de determinación, Quantikine human IGF-I, VEGF y bFGF (R \& D Systems, Inc. Minneapolis, EEUU), usando la técnica de inmunoensayo enzimático de sandwich cuantitativo (ELISA), con anticuerpo monoclonal específico para las distintas citokinas.

Análisis estadístico: se analizaron los datos con el programa estadístico SPSS v.12,0 Copyright $^{\circledR}$ 2004, SPSS Inc. SPSS Inc. Headquarters, 233 S. Wacker Drive, 11th floor, Chicago, Illinois 60606.

Los resultados obtenidos de los niveles en suero de citokinas, fueron analizados mediante la prueba $\mathrm{T}$ de Student $(\mathrm{p}<0,05)$. Se usaron curvas ROC (Receiver Operating Characteristic), de Rendimiento Predictivo o curva de Sensibilidad-Tasa de Falsos Positivos, para evaluar el rendimiento predictivo y determinar el punto de corte con el que se maximizaba la sensibilidad y especificidad de las variables predictivas estudiadas. Finalmente, usamos el análisis de regresión logística múltiple no condicional para comprobar la relación entre la tres citokinas y la aparición de ROP y proponer, en su caso un modelo predictivo. Las variables independientes que se incluyeron en el modelo fueron IGF-I, VEGF y bFGF. Se uso el método «Enter» para su inclusión, excluyéndose del modelo aquellas variables con un valor de p (estadístico de Wald) >0,10.

\section{RESULTADOS}

\section{Análisis univariante de los distintos parámetros estudiados}

\section{Peso al nacimiento}

Las medias fueron de 1.110,56 grs (DE 193,97 grs) para los enfermos, siendo ésta para los sanos pretérmino de 1.419,08 grs (DE 458,2 grs) (tabla I), la diferencia entre medias fue de 308,51 grs. Se encuentran diferencias significativas con una $\mathrm{p}<\mathrm{de}$ 0,0001 . Las curvas de rendimiento predictivo (ROC), reflejaron para este parámetro un área bajo la curva de 0,78 con una $p<$ de 0,0001 (tabla II).

\section{Edad gestacional}

Las medias fueron para el grupo de enfermos pretérmino de 29,21 semanas (DE 2,46 semanas), sien-

Tabla I. Test de la «T» de Student para los grupos estudiados

\begin{tabular}{llrr}
\hline Variable & Grupo & Media & \multicolumn{1}{c}{ DE } \\
\hline Peso al nacer & ROP & 1110,56 & 193,97 \\
\multirow{2}{*}{ Edad gestacional } & NO ROP & 1419,08 & 458,20 \\
& ROP & 29,21 & 2,46 \\
IGF -I & NO ROP & 31,32 & 2,45 \\
& ROP & 9,17 & 3,07 \\
VEGF & NO ROP & 24,07 & 7,59 \\
& ROP & 708,30 & 287,87 \\
bFGF & NO ROP & 510,81 & 150,03 \\
& ROP & 37,66 & 12,58 \\
& NO ROP & 27,94 & 11,68 \\
\hline \hline
\end{tabular}

Medias y desviaciones estándar para los distintos parámetros estudiados.

Tabla II. Valores de las áreas de las curvas ROC

\begin{tabular}{lcccc}
\hline \multirow{2}{*}{ Variable } & \multirow{2}{*}{ Área } & \multirow{2}{*}{$\mathrm{P}$} & \multicolumn{2}{c}{ IC 95\% } \\
& & & MIN & MAX \\
\hline IGF -I & 0,765 & $<0,0001$ & 0,655 & 0,874 \\
VEGF & 0,370 & 0,055 & 0,235 & 0,506 \\
bFGF & 0,439 & 0,367 & 0,307 & 0,571 \\
Peso al nacer & 0,780 & $<0,0001$ & 0,673 & 0,888 \\
Edad gestacional & 0,717 & 0,001 & 0,6 & 0,835 \\
\hline \hline
\end{tabular}

Valores de las áreas de las curvas ROC para cada parámetro estudiado. 
do para el de los sanos pretérmino de 31,32 semanas (DE 2,45 semanas) (tabla I), encontrándose una diferencia entre ambos de 2,1 semanas. Se encontraron diferencias significativas entre los dos grupos con una $\mathrm{p}<$ de 0,0001 . Las curvas ROC de sensibilidad y especificidad, reflejaron para este parámetro un área bajo la curva de 0,717 con una p de 0,001 (tabla II).

\section{Citokinas}

IGF-I (unidades en $n g / m l$ ): Las medias fueron para el grupo de enfermos pretérmino de $9,17 \mathrm{ng} / \mathrm{ml}$ (DE 3,07 ng/ml), siendo para el de los sanos pretérmino de 24,07 ng/ml (DE 7,59 ng/ml) (tabla I), encontrándose una diferencia entre ambos de 14,89 $\mathrm{ng} / \mathrm{ml}$. Se encontraron diferencias significativas entre los dos grupos con una $\mathrm{p}$ de 0,002 . Las curvas ROC de sensibilidad y especificidad, reflejaron para este parámetro un área bajo la curva de 0,765 con una $\mathrm{p}<$ de 0,0001 (tabla II) .

$V E G F$ (unidades en $\mathrm{pg} / \mathrm{ml}$ ): Las medias fueron para el grupo de enfermos pretérmino de 708,3 $\mathrm{pg} / \mathrm{ml}$ (DE 287,87 pg/ml), siendo para el de los sanos pretérmino de 510,81 pg/ml (DE 150,03 $\mathrm{pg} / \mathrm{ml}$ ) (tabla I), encontrándose una diferencia entre ambos de 197,48 pg/ml. Se encontraron diferencias significativas entre los dos grupos con una $\mathrm{p}$ de 0,03 . Las curvas ROC de sensibilidad y especificidad, reflejaron para este parámetro un área bajo la curva de 0,37 con una p de 0,055 (tabla II).

$b F G F$ (unidades en $\mathrm{pg} / \mathrm{ml}$ ): Las medias fueron para el grupo de enfermos pretérmino de 37,66 $\mathrm{pg} / \mathrm{ml}$ (DE 12,58 pg/ml), siendo para el de los sanos pretérmino de 27,94 pg/ml (DE 11,68 pg/ml) (tabla I), encontrándose una diferencia entre ambos de $9,72 \mathrm{pg} / \mathrm{ml}$. No se encontraron diferencias significativas entre los dos grupos ( $\mathrm{p}$ de 0,18 ). Las curvas ROC de sensibilidad y especificidad, reflejaron para este parámetro un área bajo la curva de 0,439 con una p de 0,367 (tabla II).

\section{Análisis multivariante (regresión logística) de las tres citokinas (IGF-I, VEGF y bFGF)}

Realizamos un análisis multivariante, que incluyó a las tres citokinas estudiadas. En éste, obtenemos significación para la IGF-I (p de 0,004), con una VEGF no significativa $(\mathrm{p}=0,074)$, objetivando que la de menor significación es la bFGF ( $\mathrm{p}=0,162)$ (tabla III). Al no obtener un modelo multivariante que englobe las tres citokinas, decidimos suprimir de dicho modelo a la bFGF, obteniéndose entonces un modelo válido sobre las dos primeras (IGF-I y VEGF). La p para la IGF-I fue de 0,006 y para la VEGF de 0,034 (tabla IV).

De este modelo deriva la siguiente fórmula, la cual nos indica en función de su resultado, cuál es el riesgo de padecer retinopatía, dependiendo de los niveles en suero de la IGF-I y VEGF:

$$
\mathrm{p}(\text { enfermo })=\frac{1}{1+\exp [-(-0,242+(0,048 \mathrm{xIGF})+(0,002 \mathrm{xVEGF}))]}
$$

\section{DISCUSIÓN}

En el año 1984, aparece la clasificación internacional de la retinopatía de la prematuridad (ICROP), producto del consenso de un grupo internacional de expertos sobre la ROP. Esta clasificación fue ampliada y revisada en el año 1987 (12). Posteriormente, en 1988 aparece publicado el primer informe con resultados preliminares del grupo cooperativo CRYO-ROP (13). Este estudio, constituyó un hito en la retinopatía de la prematuridad, mediante los criterios de indicación para su tratamiento (14). Con el tiempo, la Academia Americana de Oftalmología (AAO), ha ido desarrollando nuevas y mejores pautas para unificar criterios a

Tabla III. Análisis multivariante

\begin{tabular}{lcccc}
\hline & \multirow{2}{*}{ P } & OR & \multicolumn{2}{c}{ IC 95\% } \\
& & & MIN & MAX \\
\hline IGF-I & 0,004 & 1,053 & 1,017 & 1,091 \\
VEGF & 0,074 & 0,999 & 0,997 & 1,000 \\
bFGF & 0,162 & 0,986 & 0,966 & 1,006 \\
Constante & 0,370 & 1,639 & - & - \\
\hline \hline
\end{tabular}

Análisis multivariante para las tres citokinas.

Tabla IV. Modelo final del análisis multivariante

\begin{tabular}{lcccc}
\hline & \multirow{2}{*}{ P } & OR & \multicolumn{2}{c}{ IC 95\% } \\
& & & MIN & MAX \\
\hline IGF-I & 0,006 & 0,954 & 0,922 & 0,986 \\
VEGF & 0,034 & 1,002 & 1,000 & 1,003 \\
Constante & 0,635 & 0,785 & - & Constante \\
\hline \hline
\end{tabular}

Modelo final del análisis multivariante en base a la IGF-I Y VEGF. 
nivel global, y lograr que el diagnóstico y tratamiento de la ROP sea cada vez mejor $(10,11)$.

Este desarrollo en el conocimiento de la enfermedad, ha hecho que en los últimos tiempos, grupos de trabajo independientes de diversos países, comiencen a cuestionar los criterios dados por la AAO $(15,16)$, e incluso diseñan los suyos propios para identificar a los niños a los que se deben proponer revisiones de fondo de ojo $(17,18)$. Estas disquisiciones, surgen de problemas encontrados con la elegibilidad de dichos criterios para el despistaje de la enfermedad, dado que estos en numerosas ocasiones hacen que pacientes que no padecen ROP sean examinados y pacientes enfermos escapen al despistaje, con las consiguientes secuelas del no tratamiento de estos enfermos (19.) No hay que minimizar la importancia que tiene que un paciente que no padezca ROP, sufra los efectos de la exploración, ya que no es infrecuente el encontrar complicaciones desde el inicio de la preparación de la exploración de prematuros, como las derivadas de la instilación de midriáticos o el gran stress derivado de la exploración oftalmológica $(20,21)$.

El objetivo de un buen sistema de despistaje para la ROP debe tener en cuenta qué niños padecen enfermedad retiniana y de estos cuáles van a desarrollar grados tratables de enfermedad. De esta manera no se realizarían exploraciones a pacientes que no las necesitasen y dicha exploración iría encaminada a diagnosticar grados umbrales de la ROP. Así se ahorrarían recursos, complicaciones derivadas de la exploración y tiempo en el diagnóstico. Algunas publicaciones incluso comentan el no realizar exploraciones de seguimiento a pacientes con grados leves, que de manera habitual tienden a la regresión espontánea $(13,19)$. En este estudio encontramos que tanto la EG, como el PN, son factores de riesgo de igual capacidad predictora de enfermedad que la IGF-I y la VEGF. Estas como hemos expuesto en los resultados, resultaron significativas la IGF-I, y la VEGF no así el bFGF, aunque con una p de 0,18 , lo que nos hace sospechar en un posible error beta, es decir, en una falta de potencia estadística debido al tamaño muestral de nuestro estudio. A pesar de esto, se han expuesto casos en los que estas citokinas podrían servir como factores de seguimiento en el tratamiento de la ROP (22).

Según las curvas ROC de predicción de sensibilidad de pruebas diagnósticas, nos presentan unas tablas en las que se proponen valores como puntos de corte en los que obtendríamos una u otra sensi- bilidad de discriminación entre sanos y enfermos. Estas, indican que la IGF-I, es un factor protector de la enfermedad y que el VEGF es un factor de riesgo. Nosotros resaltamos en la tabla V, valores intermedios donde más se aproximan la sensibilidad y la especificidad de cada parámetro. Es Hellström en el 2003 el que establece valores de IGF-I en pacientes sin ROP en $33 \mu \mathrm{g} / \mathrm{l}$, encontrándose en nuestro estudio una media para este grupo de $24,07 \mathrm{ng} / \mathrm{ml}(\mu \mathrm{g} / \mathrm{l}$ $=\mathrm{ng} / \mathrm{ml}$ ), para una muestra de 84 pacientes, similar a la nuestra con 74 (2).

En el análisis multivariante, resulta un modelo predictivo válido aquel que incluye la IGF-I y la VEGF en la fórmula que hemos expuesto anteriormente. $\mathrm{Al}$ sustituir en dicha fórmula valores para una IGF-I y VEGF de un paciente supuestamente enfermo según los datos emitidos por las curvas ROC (IGF-I= 5 $\mathrm{ng} / \mathrm{ml}$ y $\mathrm{VEGF}=1.000 \mathrm{pg} / \mathrm{ml}$ ) resulta una probabilidad de padecer ROP del $82 \%$. Por el contrario, al sustituir estos valores por los de un paciente supuestamente sano según los datos emitidos por las curvas ROC, (IGF-I $=25 \mathrm{ng} / \mathrm{ml}$ y VEGF $=250 \mathrm{pg} / \mathrm{ml}$ ) resulta una probabilidad de padecer ROP del $28 \%$.

Con esto, podemos concluir que estos dos mitógenos pueden servir como indicadores de alta sensibilidad en el screening de la ROP y que el modelo anterior puede ser válido para proponer con exac-

Tabla V. Valores de sensibilidad y especificidad

\begin{tabular}{lrcc}
\hline Variable & Valores & $\begin{array}{c}\text { Sensibilidad } \\
(\%)\end{array}$ & $\begin{array}{c}\text { Especificidad } \\
(\%)\end{array}$ \\
\hline IGF -I & & 100 & 18,9 \\
& 0,91 & 70,3 & 70,3 \\
VEGF & 6,22 & 27 & 91,9 \\
& 27,30 & 100 & 5,4 \\
& 173,23 & 45,9 & 32,4 \\
& 467,28 & 32,4 & 32,4 \\
bFGF & 517,64 & 21,6 & 43,2 \\
& 580,17 & 10,8 & 78,4 \\
& 1002,92 & 100 & 0 \\
& 1,25 & 48,6 & 43,2 \\
Peso al nacer & 22,93 & 43,2 & 43,2 \\
& 24,24 & 29,7 & 64,9 \\
& 31,77 & 10,8 & 86,5 \\
& 58,97 & 100 & 2,7 \\
Edad Gestacional & 717 & 78,4 & 47,6 \\
& 1107,5 & 70,3 & 70,3 \\
& 1205 & 48,6 & 89,2 \\
& 1413,5 & 51,4 & 5,4 \\
& 25,5 & 100 & 59,5 \\
& 29,5 & 81,1 & 67,6 \\
\hline \hline
\end{tabular}

Valores de sensibilidad y especificidad para los diferentes parámetros, generados por las curvas ROC. 
titud a los niños susceptibles de este despistaje en función de la IGF-I y la VEGF. También planteamos la posibilidad de usar estos factores de crecimiento como indicadores de la gravedad de la ROP. Véase por ejemplo estudios que apuntan la posible relación entre la ablación de la retina con crioterapia o láser en pacientes con ROP tratable, y los niveles de IGF-I, VEGF y bFGF, para el seguimiento de la efectividad de estos tratamientos en relación con la gravedad de la enfermedad (22).

Será necesario sin embargo, proseguir con esta línea de investigación a mayor escala y ampliando el número de muestra de nuestros estudios. De poder demostrar con mayor muestra y con el consenso de otros centros que esto fuera así, se podría aplicar este modelo no sólo a la ROP, sino también, como diagnóstico precoz para otras enfermedades con similar etiopatogenia, como la retinopatía diabética, la degeneración macular asociada a la edad, e incluso a enfermedades de origen neovascular del tipo de ciertos tumores.

Es entonces nuestra intención el progresar, no solo en el conocimiento más profundo de esta enfermedad, sino también en el diagnóstico certero del momento en el que se produce, para poder actuar con el tratamiento necesario antes de que las secuelas de esta sean irreversibles y evitemos así las consecuencias de esta enfermedad.

\section{BIBLIOGRAFÍA}

1. Neely KA, Gardner TW. Ocular neovascularization: clarifying complex interactions. Am J Pathol 1998; 153: 665670.

2. Hellstrom A, Engstrom E, Hard AL, Albertsson-Wikland $K$, Carlsson B, Niklasson A, et al. Postnatal serum insulinlike growth factor I deficiency is associated with retinopathy of prematurity and other complications of premature birth. Pediatrics 2003; 112: 1016-1020.

3. Villegas Becerril E, Fernández Molina F, Gonzalez R, Gallardo Galera JM. Valores de IGF-I séricos en la ROP. Buscando nuevas indicaciones para su screening. Arch Soc Esp Oftalmol 2005; 80: 233-238.

4. Mesiano S, Ferrara N, Jaffe RB. Role of vascular endothelial growth factor in ovarian cancer: inhibition of ascites formation by immunoneutralization. Am J Pathol 1998; 153: 1249-1256.

5. Jakeman LB, Armanini M, Phillips HS, Ferrara N. Developmental expression of binding sites and messenger ribonucleic acid for vascular endothelial growth factor suggests a role for this protein in vasculogenesis and angiogenesis. Endocrinology 1993; 133: 848-859.

6. McColm JR, Geisen P, Hartnett ME. VEGF isoforms and their expression after a single episode of hypoxia or repeated fluctuations between hyperoxia and hypoxia: relevance to clinical ROP. Mol Vis 2004; 10: 512-520.
7. Ozaki H, Okamoto N, Ortega S, Chang M, Ozaki K, Sadda S, et al. Basic fibroblast growth factor is neither necessary nor sufficient for the development of retinal neovascularization. Am J Pathol 1998; 153: 757-765.

8. Boulton M, Gregor Z, McLeod D, Charteris D, JarvisEvans J, Moriarty $P$, et al. Intravitreal growth factors in proliferative diabetic retinopathy: correlation with neovascular activity and glycaemic management. Br J Ophthalmol 1997; 81: 228-233.

9. Lashkari K, Hirose T, Yazdany J, McMeel JW, Kazlauskas A, Rahimi N. Vascular endothelial growth factor and hepatocyte growth factor levels are differentially elevated in patients with advanced retinopathy of prematurity. Am J Pathol 2000; 156: 1337-1344.

10. American Academy of Pediatrics. Section on Ophthalmology. Screening examination of premature infants for retinopathy of prematurity. Pediatrics 2001; 108: 809-811.

11. Section on Ophthalmology American Academy of Pediatrics; American Academy of Ophthalmology; American Association for Pediatrics Ophthalmology and Strabismus. Screening examination of premature infants for retinopathy of prematurity. Pediatrics 2006; 117: 572-576.

12. Palmer EA, Flynn JT, Hardy RJ, Phelps DL, Phillips CL, Schaffer DB, et al. Incidence and early course of retinopathy of prematurity. The Cryotherapy for Retinopathy of Prematurity Cooperative Group. Ophthalmology 1991; 98: 1628-1640.

13. de la Cruz Bertolo FJ, Pallas Alonso CR, Tejada Palacios $P$. Screening for retinopathy of prematurity: reliability. An Esp Pediatr 1999; 50: 156-160.

14. Good WV; Early Treatment for Retinopathy of Prematurity Cooperative Group. Final results of the Early Treatment for Retinopathy of Prematurity (ETROP) randomized trial. Trans Am Ophthalmol Soc 2004; 102: 233-248.

15. Schulenburg WE, Bloom PA. Current problems in the management of ROP. Acta Ophthalmol Scand Suppl 1995; 214: 14-16.

16. Goble RR, Jones HS, Fielder AR. Are we screening too many babies for retinopathy of prematurity? Eye 1997; 11: 509-514.

17. Fielder AR, Haines L, Scrivener R, Wilkinson AR, Pollock JI; Royal Colleges of Ophthalmologists and Paediatrics and Child Health, and the British Association of Perinatal Medicine. Retinopathy of prematurity in the UK II: audit of national guidelines for screening and treatment. Eye 2002; 16: 285-291.

18. Hutchinson AK, Saunders RA, O'Neil JW, Lovering A, Wilson ME. Timing of initial screening examinations for retinopathy of prematurity. Arch Ophthalmol 1998; 116: 608-612.

19. Pallás Alonso CR, Tejada Palacios P, Medina López MC, Martín Puerto MJ, Orbea Gallardo C, Barrio Andrés MC. Retinopatía del prematuro: Nuestra experiencia. An Esp Pediatr 1995; 42: 52-56.

20. Slevin M, Murphy JF, Daly L, O'Keefe M. Retinopathy of prematurity screening, stress related responses, the role of nesting. Br J Ophthalmol 1997; 81: 762-764.

21. Rush R, Rush S, Nicolau J, Chapman K, Naqvi M. Systemic manifestations in response to mydriasis and physical examination during screening for retinopathy of prematurity. Retina 2004; 24: 242-245.

22. Villegas Becerril E, Gonzalez Fernandez R, Fernandez Molina F, Gallardo Galera JM. Growth factor levels and ROP. Ophthalmology 2005; 112: 2238. 